\title{
Making mHealth Work for All
}

Information and Communication technologies (ICTs) are going to have a major impact on health and health systems in the near future. To ensure that everyone (including the very poor) benefits from this new technology, it is vital that those designing new technologies, people responsible for health systems and social researchers come together to explore the opportunities and potential challenges, especially for low- and middle-income countries (LMICs).

Rapid increases in access to mobile phones and the internet in LMICs countries have stimulated interest in the potential contribution that ICTs can make towards increasing access to safe, affordable and effective health services. This has encouraged investment in the development of applications of these technologies. At the same time, governments and international agencies have prioritised measures aimed at making progress towards universal health coverage (UHC) and the new sustainable development goals (SDGs), by strengthening health systems. There has, however, been insufficient communication between researchers and innovators working on these parallel issues.

\section{Sharing knowledge at the Fourth Global Symposium on Health Systems Research}

Health Systems Global organises a biennial symposium for the international community of health system researchers. More than 2,000 people participated in the Fourth Global Symposium on Health Systems Research (HSR2106) in Vancouver in November 2016. Its high-profile plenary discussions paid little attention to ICTs and their role in transforming health systems, although several parallel sessions and posters addressed interventions for frontline health workers, better health care, community based family planning; mHealth and health system resilience; and skills-building in mHealth. This report is based on one of the parallel sessions, and a brainstorming discussion, which the Impact Initiative supported with the aim of stimulating further dialogue between the $\mathrm{mHealth}$, health systems and social science research communities.

In one of the parallel sessions, grantees from two ESRC-DFID funded projects presented findings about the use of mobile phones for seeking health information in LMICs.

Based on research from ' $I$ CTs and The Changing Health Knowledge Economy: How People Find Health Information in Bangladesh' Sabrina Rasheed reported high levels of phone ownership and use in Bangladesh, but substantial differences in use between rich and poor households and between men and women. Despite a number of innovations using ICTs - such as sending health-related SMS messages to mobile phones and providing access to health information through medical help lines or the internet - only a small proportion of questionnaire survey respondents had used their mobile phone to find health information.

Simon Mariwah and Kate Hampshire referred their project on 'mobile phone use among children and young adults in Ghana, Malawi and South Africa'. They found increasing use of the internet as a source of health information in the context of weak health systems that did not meet the needs of the young. They also found that community health workers were using their phones to call patients, colleagues and community leaders and to learn about "modern" treatments on the internet.

The discussant, Alain Labrique, reviewed a decade of country-level and government experiences in leveraging mobile technologies to alleviate health system constraints and emphasised the need to move beyond pilot testing of interventions to the implementation of evidence-based strategies for integrating ICTs into health systems and government health services, at scale. He illustrated how, in several countries, the falling cost of mobile phones and ICTs, combined with the rapid pace of technological development and the focus on supplyside challenges, is opening up major opportunities for improving the performance of these services.

In addition, during HSR2016, a group of people with different disciplinary approaches and experiences met to discuss the opportunities and challenges associated with mHealth. Everyone agreed that ICTs were likely to have a major impact on the health systems of the future, but they had different visions of the likely pathways of development. 
Participants in the discussion of mHealth opportunities and challenges, HSR2016, 17 November 2016

\begin{tabular}{|l|l|}
\hline Name & Institution \\
\hline Gerry Bloom & Institute of Development Studies, UK \\
\hline Carl Leitner & IntraHealth, USA \\
\hline Alain Labrique & Johns Hopkins Bloomberg School of Public Health, USA \\
\hline Dykki Settle & PATH, USA \\
\hline Melinda Moree & Vital Wave, USA \\
\hline Simon Mariwah & UCC, Ghana \\
\hline Kate Hampshire & Durham University, UK \\
\hline Tom Feeny & R4D, USA \\
\hline Linda Waldman & Institute of Development Studies, UK \\
\hline Kati Wilkins & Women in Global Health, US \\
\hline
\end{tabular}

\section{The potential role of ICTs in changing the performance of government health systems}

The participants of the discussion, involved in developing $\mathrm{mHealth}$ innovations emphasised the potential role of ICTs in changing the performance of government health systems. They pointed out that investments by donor agencies and foundations are increasingly providing support for the creation of national health information platforms. Moreover, plans for extending facility-based data capture and service delivery reporting systems to the community, through investing in patient-based information systems, are being developed. Ministries of health in many African and South Asian countries are using ICTs and mobile technologies to monitor the performance of health services and specific public health programs. They argued that ICTs have the potential to become an important driver for change, by enabling governments to obtain timely health system performance data, monitor and respond to supply-side challenges and test demandcreation and public information strategies through automated voice and text messages. They outlined a future vision of an ICT-enabled government health system that uses limited resources efficiently to meet the needs of its population.

The social scientists and others who were less involved with the technological side and more connected with users, focused on the pluralistic nature of health systems, in which people seek advice and treatment from a variety of providers, both formal and informal, medically-trained and/or relying on other healing techniques and either integrated into, or excluded from, the regulatory system. They emphasised the degree to which relatively poor people seek care for common health problems outside the public health system and the way that this reliance of all social categories of people on non-state providers is creating new markets for health information, drugs and diagnostic devices. They also emphasised the role of investment by the private sector in systems for the distribution and sale of drugs and diagnostic services and in the development of poorlyregulated health information platforms. They argued that this is likely to lead to lead to a variety of undesirable outcomes in the absence of strong and coherent government leadership and citizen protections.

What are the priorities for investment to improve the performance of health systems?

All participants in the discussion agreed that the pace of change was likely to accelerate. They also agreed that governments, international agencies and global governance arrangements will have an important influence on the development of ICT-enabled health systems. There were, however, different views on the priorities for investment aimed at improving the performance of health systems in meeting the needs of the poor across diverse settings and contexts. This has important implications for further research.

One view began with the important role that adequately financed and well-managed government health services can play in providing access to health services, including point-of-care diagnoses and appropriate drugs.

Many LMICs have established ICT-based platforms and systems, with varying degrees of scale and functionality. Donor agencies and governments will need to make substantial investments to strengthen these platforms. The experiences of a number of countries have underlined the importance of strong leadership to ensure that investments in information systems are made in robust, standards-based technologies that reduce, rather than exacerbate, inequities. This leadership is vital to ensure that data are used for management decisions, and not simply for periodic reporting of health system indicators. Several people emphasised the need to ensure that investments in disease-specific information systems ensure strong government support with commitments to avoid the creation of parallel systems. Safeguarding of information was also raised as a key concern given the potential use of patient-based information for political and non-health purposes. 
The second view emphasised the need to understand the context within which new technologies are being incorporated.

There are large differences between countries in terms of the public sectors' management capacity and the degree to which governments are committed to meeting the needs of the entire population, including the poor. Complex health markets, in the context of pluralistic health systems in LMICs, already involve a wide variety of state and non-state providers of medical care and drugs. Mobile phone operators and companies with internet platforms are establishing a growing presence in these countries. There is evidence that some of their citizens (especially the young) are trying these new sources of medical information and advice as an alternative to government health systems with which they are dissatisfied. The discussion identified a number of risks associated with the quality of information that is made available and the possibility of capture by stakeholders with a financial interest in encouraging greater use of diagnostic devices and drugs.

Government and donor agencies can play an important role in stimulating innovations likely to benefit the poor and creating regulatory arrangements to protect the interests of the public, especially when the perspectives of the poor are taken into account. One area for potential investment would be in producing and making widely available treatment guidelines for common illnesses and in creating innovative partnerships between different organisations (public and private) to increase access to appropriately-vetted health information.

\section{Bringing together researchers and innovators to explore and respond to opportunities and challenges}

Participants in the discussion emphasised the speed of technological change and the growing levels of public and private sector investments in various forms of $\mathrm{mHealth}$. They agreed on the potential role of government health services and also on the likelihood of rapid changes in markets for health services. As discussion deepened, the existence of multiple challenges and necessary considerations for prudent digital health system strengthening became clear.

In sharing these different visions of pathways of development, participants also became aware of the different implications for investment strategies in innovation, the different ways of evaluating appropriate regulatory arrangements and the need to bring the supply-side challenges together with the users' experiences in order for these investments to yield the most benefit whilst minimising the risks of harm.

The convening of these interdisciplinary discussions to share research findings, explore the opportunities associated with the rapid development and spread of information technologies and examine the regulatory challenges likely to emerge, is vital to ensure that everyone, and especially the very poor in LMIC, benefits.

Credits 